\title{
Outcomes of Antiretroviral Therapy in the Swiss HIV Cohort Study: Latent Class Analysis
}

\author{
Olivia Keiser · Ben Spycher • Andri Rauch • Alexandra Calmy • \\ Matthias Cavassini - Tracy R. Glass · Dunja Nicca • \\ Bruno Ledergerber - Matthias Egger - The Swiss HIV Cohort Study
}

Published online: 1 June 2011

(C) Springer Science+Business Media, LLC 2011

\begin{abstract}
An in-depth understanding of the different groups that make up the HIV-infected population should inform prevention and care. Using latent class analysis (LCA) we identified seven groups with similar sociodemographic and behavioral characteristics at enrolment in the Swiss HIV Cohort Study: older gay men, younger gay men, older heterosexual men, injection drug users, single migrants, migrant women in partnerships and heterosexual men and women. Outcomes of combination antiretroviral therapy (ART) were analyzed in 1,633 patients starting ART. Compared to older gay men, the probability of a virologic response to ART was reduced in single migrants, in older heterosexual men and in IDUs. Loss to follow-up was higher in single migrants and IDUs, and mortality was increased in older heterosexual men and IDUs. Sociobehavioral groups identified by LCA allow insights above
\end{abstract}

Olivia Keiser and Ben Spycher contributed equally to this study. The members of The Swiss HIV Cohort Study are given in the acknowledgement.

The work was presented at the Conference of Retroviruses and Opportunistic Infections (CROI 2010) in San Francisco.

O. Keiser $(\square) \cdot$ B. Spycher $\cdot$ M. Egger

Institute of Social and Preventive Medicine (ISPM), University

of Bern, Finkenhubelweg 11, 3012 Bern, Switzerland

e-mail: okeiser@ipsm.unibe.ch

A. Rauch

University Clinic for Infectious Diseases, Bern University

Hospital and University of Bern, Bern, Switzerland

A. Calmy

Division of Infectious Diseases, University Hospital Geneva, Geneva, Switzerland

M. Cavassini

Division of Infectious Diseases, University Hospital Lausanne,

Lausanne, Switzerland what can be gleaned from traditional transmission groups, and may identify patients who could benefit from targeted interventions.

Keywords HIV infection · Antiretroviral therapy · Disease progression $\cdot$ Mortality $\cdot$ Social factors $\cdot$ Behavior . Latent class analysis

\section{Introduction}

Highly active antiretroviral therapy (ART) has substantially reduced morbidity and mortality in HIV-infected patients [1]. However, mortality continues to be increased compared to the general population in many patient groups $[2,3]$, treatment fails in a substantial minority of patients and loss to follow-up is common [4]. Social and behavioral factors are important predictors of health outcomes [5]. In HIV infection socio-demographic and behavioral characteristics of patients have repeatedly been shown to be

T. R. Glass

Basel Institute for Clinical Epidemiology and Biostatistics,

University Hospital Basel, Basel, Switzerland

D. Nicca

Division of Infectious Diseases, Cantonal Hospital St.Gallen,

St.Gallen, Switzerland

B. Ledergerber

Division of Infectious Diseases and Hospital Epidemiology,

University Hospital, University of Zürich, Zürich, Switzerland 
associated with late diagnosis of the infection, and with progression of the disease. For example, a collaborative analysis of European and North American cohort studies showed that mortality was lowest in gay men, somewhat higher in heterosexually infected individuals and much higher in people infected through injection drug use (IDU) [3]. In contrast, a Canadian study found that mortality was similar in IDUs and non-IDUs [6].

Previous studies generally focused on the main HIV transmission groups or identified single socio-demographic and behavioral characteristics that are independently associated with the outcomes of interest. However, the transmission groups consist of heterogeneous groups of patients, including, for example, younger immigrants and older Swiss patients who were infected heterosexually, current and former IDUs, and young and older gay men. Age is associated with adherence to ART [7] and there may be synergistic effects between immune deficiency and chronic immune activation, which accelerates ageing $[8,9]$. It is therefore likely that analyses of outcomes of ART based on broad transmission groups will hide differences within groups, and that the different composition of transmission groups might explain conflicting results between such studies [3, 6].

Studies that examined socioeconomic position (SEP) and treatment outcomes have also found conflicting results. Low SEP, poverty and minority ethnic groups were associated with poorer prognosis $[10,11]$ but some studies found no relationship [12,13] or associations in some groups only, for example in women [14]. It has been suggested that a possible reason for these conflicting results may be that SEP is mediated by a variety of factors that differ between settings and that single measures of SEP, for example income, and proxy measures such as ethnicity or injection drug use, are insufficient to reliably capture SEP in different populations and settings [15].

An alternative approach to analyzing differences between transmission groups or levels of SEP is to examine how relevant variables cluster across patients. Latent class analysis (LCA) can be used for this purpose [16]. Originally developed in the social sciences, LCA is increasingly used in medical research, for example to identify disease phenotypes $[17,18]$ or to evaluate diagnostic tests in the absence of a gold standard [19]. In the present study we used LCA to examine the following hypotheses in the Swiss HIV Cohort Study (SHCS), a large prospective cohort that is representative of persons infected with HIV in Switzerland [20]: (1) meaningful, distinct socio-demographic and behavioral groups can be identified in the SHCS through LCA; (2) these groups differ from the main HIV transmission groups and include groups that are not directly observable using a single variable approach; (3) group membership is associated with relevant treatment variables (such as late initiation of ART or adherence) and with treatment outcomes.

\section{Methods}

\section{The Swiss HIV Cohort Study (SHCS)}

The SHCS was established in 1988 as a nationwide, prospective cohort study of HIV-infected participants who are followed at one of seven study centers based at University or Cantonal hospitals, or at a private practice or regional hospital associated with a study center [20, 21]. A standardized questionnaire is used for data collection which is completed by the treating physician. Socio-demographic and behavioral data are recorded at enrolment (i.e., year of birth, gender, nationality, ethnicity, education, profession, region of origin, income, HIV test result, sexual orientation, presumed mode of HIV transmission). At each semiannual visit further socio-economic and behavioral data are collected, including source of income, ability to work, current IDU, partnerships and condom use. Antiretroviral treatment, clinical events and hospitalizations are documented in detail. Completed questionnaires are sent to the SHCS data centre where the data are entered and quality checks are performed.

Transmission groups include gay men, heterosexually infected patients, IDU, patients infected via blood products and patients with unknown route of infection. HIV-1 RNA is determined using the Roche Amplicor HIV-1 Monitor or the Cobas-Taqman assay. Clinical AIDS diagnoses (Centers for Disease Control and Prevention [CDC] stage C) are recorded by the treating physicians on the basis of the 1993 CDC criteria [22]. Hepatitis B (HBV) co-infection is defined by a positive $\mathrm{HBV}$ surface antigen ( $\mathrm{HBsAg}$ ) and hepatitis $\mathrm{C}(\mathrm{HCV})$ infection by a positive anti-HCV antibody test. Laboratory results are transferred automatically from laboratories to the SHCS data centre. Data quality is regularly checked by the data centre and a system with incentives rewarding high quality of data is in place.

At all sites institutional review boards approved the collection of data. All study participants provided informed consent.

\section{Eligibility Criteria and Definitions}

All participants newly enrolled in the Swiss HIV Cohort Study between 1st of January 2000 and August 2008 were included in the LCA to identify socio-behavioral groups. Participants who were treatment-naïve and started ART at or after enrolment were included in the analysis of treatment outcomes. The closing date of the database for the 
present analysis was August 2008. ART was defined as combination antiretroviral therapy consisting of at least three drugs from at least two drug classes. Participants who reported IDU within the last 6 months prior to registration were classified as 'current IDU'. All other participants who reported IDU prior to registration were classified as 'former IDU'.

\section{Latent Class Analysis (LCA)}

We used LCA [16] to identify groups of individuals with similar socio-demographic and behavioral characteristics at enrolment into the cohort. The method assumes that the population is composed of subpopulations (latent classes), which are not directly observed but inferred from the observed characteristics of individuals. Within each class, the variables are assumed to be independent, i.e., all associations between variables are assumed to arise from the presence of different latent classes and within each class heterogeneity is reduced. All relevant sociodemographic and behavioral variables were included in the LCA. Two authors (OK and BS) selected the variables and both the selection of variables and the definitions of categories were done a priori and not modified later on. We included the following variables: sex, age $(<25,25-34$, $35-44,45-54, \geq 55$ years), region of origin (Switzerland and North-West Europe, Southern Europe, Sub-Saharan Africa, Latin America, Asia/Eastern Europe, Other), profession (self employed, apprentice/trainee, higher management, middle management, employee, houseman/wife, student), main source of income (employment, family support, unemployment benefit or other insurance), sexual orientation (gay, bisexual, heterosexual), partner (stable partner only, stable and occasional partners, occasional partners only, no partner), and history of IDU (never, former, current).

The models were fitted using Mplus software (Muthen \& Muthen, Los Angeles, CA, USA). The appropriate number of classes was selected using the Bayesian information criterion (BIC) [23]. Probabilities of belonging to the different latent classes of a fitted model can be computed for each individual based on his/her socio-demographic and behavioral characteristics. Individuals were allocated to the groups for which they had the highest membership probability [23].

\section{Outcomes}

Four outcomes were considered: virologic response, loss to follow-up, all cause mortality and adherence to ART. Virologic response was defined as suppression of HIV-1 viral load to $<50$ copies/ml at 6 months after starting ART among patients who were followed for at least 6 months.
The value closest to 6 months (within 5-12 months) after start of ART was used. A patient was considered lost to follow-up if the last visit was more than 14 months before the closing date of the database and the patient was not known to have died. Only participants who potentially had 14 months of follow-up were included in the analysis of loss from follow-up. Adherence had been assessed since May 2003 by asking two questions at every visit: (1) How often did you miss a dose in the last four weeks (every day, more than once a week, once a week, once every second week, once a month, never), (2) Did you not take drugs for $>24 \mathrm{~h}$ in the last four weeks? (yes or no). Good adherence was defined as having missed no dose during the four weeks preceding the most recent study visit.

\section{Statistical Analysis}

We used univariable and multivariable Cox regression for time-to-event analyses of mortality and loss to follow-up and logistic regression for virologic response and adherence. Time was measured from the start of treatment to the date the endpoints occurred, or the date of the last follow-up visit. Models were adjusted for values at the start of ART: age, CD4 cell count $(0-49,50-99,100-199,200-349, \geq 350$ cells/ $\mu$ l), viral load (<50-9,999, 10,000-99,999, $\geq 100,000$ copies/ml), clinical stage (CDC stage A and B, or C) and type of ART regimen (PI-based, NNRTI-based, other). Some analyses were additionally adjusted for sex.

Missing values of CD4 cell counts and viral load at the start of ART were imputed on the basis of whether the patient died, age, sex, year of starting ART and CDC stage of disease. In these imputations, values of the missing data were randomly sampled from their predicted distributions. Analyses were run on each of 20 datasets, and the results combined with Rubin's rules [24].

Analyses were done using Stata software (version 10, Stata Corporation, College Station, Texas). Results are presented as odds ratios (OR) or hazard ratios (HR).

\section{Results}

A total of 4,483 participants were included in the LCA. The majority of participants $(3,063 ; 68.3 \%)$ were men, median age was 37 years (IQR 31-43 years) and the median CD4 cell count at enrolment was 359 cells/ $\mu$ l (IQR 206-540). Distribution across groups of presumed transmission was as follows: heterosexual (1,991 participants; $44.4 \%)$, gay men $(1,680 ; 37.5 \%)$, IDU $(598 ; 13.3 \%)$ and other or unknown $(214 ; 4.8 \%)$. A total of 1,633 participants (36.4\%) starting ART were included in mortality analyses, with a median duration of follow-up of 2.8 years (IQR 1.1-5.0 years). The remaining participants died or were 
Fig. 1 Inclusion of participants into different analyses

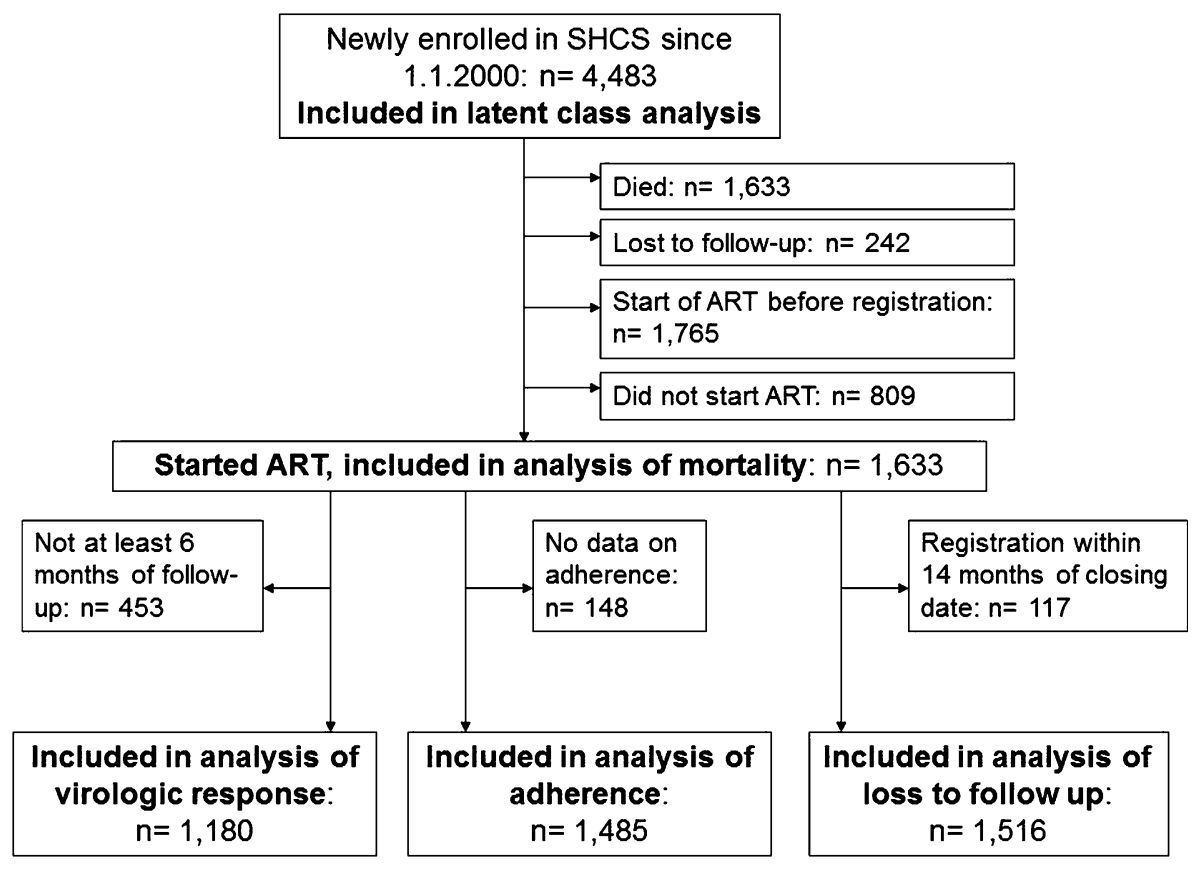

lost to follow-up before starting ART, started ART before enrolment or were still under follow-up without having started ART (Fig. 1). Missing baseline values of CD4 cell count were imputed in 163 patients $(10.0 \%)$, and missing viral load values in 340 patients $(20.1 \%)$. The 2,850 patients who were excluded from the analysis of treatment outcomes were more likely to be female (32.9 vs. $29.6 \%$ ), more likely to have a history of IDU (17.9 vs. $13.0 \%$, $P<0.001)$, less likely to have a homosexual orientation (32.8 vs. $39.7 \%, P=0.03$ ) and more likely to live without external financial support (62.3 vs. $52.9 \%, P<0.001)$.

A total of 1,516 participants were included in the analysis of loss to follow-up: 117 participants $(7.2 \%)$ were excluded because they were enrolled within 14 months before the closing date. Overall 1,485 patients were included in the analysis of adherence to ART: $148(9.1 \%)$ were excluded because they did not have data on adherence. Finally, 1,180 patients were included in the analysis of virologic response: 453 participants $(27.7 \%)$ were excluded because they did not have at least 6 months of follow-up (Fig. 1).

Socio-Behavioral Groups Identified by Latent Class Analysis

A latent class model with seven classes was chosen, based on the lower BIC value in this model compared to models with fewer or more classes. Fig. 2 shows the formation of the groups and the decrease in BIC values as the number of classes increased. The group of older gay and bisexual men was identified early on (G1 in model 2) and present in all subsequent models, with some attrition to other groups. The third group (G3) in model 3 included single migrants and migrant women in stable partnership whereas the second group (G2) later split into IDUs, heterosexual men and women, and older heterosexual men. For 2,791 $(62.3 \%)$ participants, the probability of belonging to the group they were assigned to was $\geq 0.90$, while for 433 $(9.7 \%)$ participants this probability was $<0.60$, indicating more ambiguous membership. The median probability ranged from 0.82 (IQR $0.63-0.92$ ) in the group of heterosexual men and women to $0.99(0.95-1.00)$ in IDUs.

The distribution of the variables included in the latent class analysis across the seven groups is shown in Table 1 and Box 1 gives a summary description of the groups. All socio-behavioral groups were dominated by one transmission group. For example, participants presumably infected through heterosexual contacts dominated four socio-behavioral groups: heterosexual men and women (729/884; $82.5 \%)$, older heterosexual men $(298 / 431 ; 69.1 \%)$, single migrants $(403 / 444 ; 90.8 \%)$ and migrant women in partnership $(356 / 411 ; 86.6 \%)$. The group of heterosexual men and women included some individuals presumably infected through IDU $(66 / 884 ; 7.5 \%)$. All were former IDUs. Also, the group described as older heterosexual men included a minority $(96 / 431 ; 22.3 \%)$ of gay men. The six women who were classified into the two groups of 'gay men' were all lesbian women.

\section{Characteristics at the Start of ART}

CD4 cell count, clinical stage and prevalence of HCV and HBV infection at start of ART are shown in Table 2. The median CD 4 count ranged from 175 cells/ $\mu$ l in heterosexual men and women to 225 cells/ $\mu$ l in migrant women 
Fig. 2 Stepwise identification of socio-behavioral groups in latent class analysis. The size of shaded boxes is proportional to the percentage of the 4,483 participants allocated to the group. A model with seven groups had the lowest BIC (Bayesian Information Criterion): in a model with eight groups BIC increased to 64,112 . The seven groups of the final model were assigned labels according to their most pertinent characteristics (see Box 1).

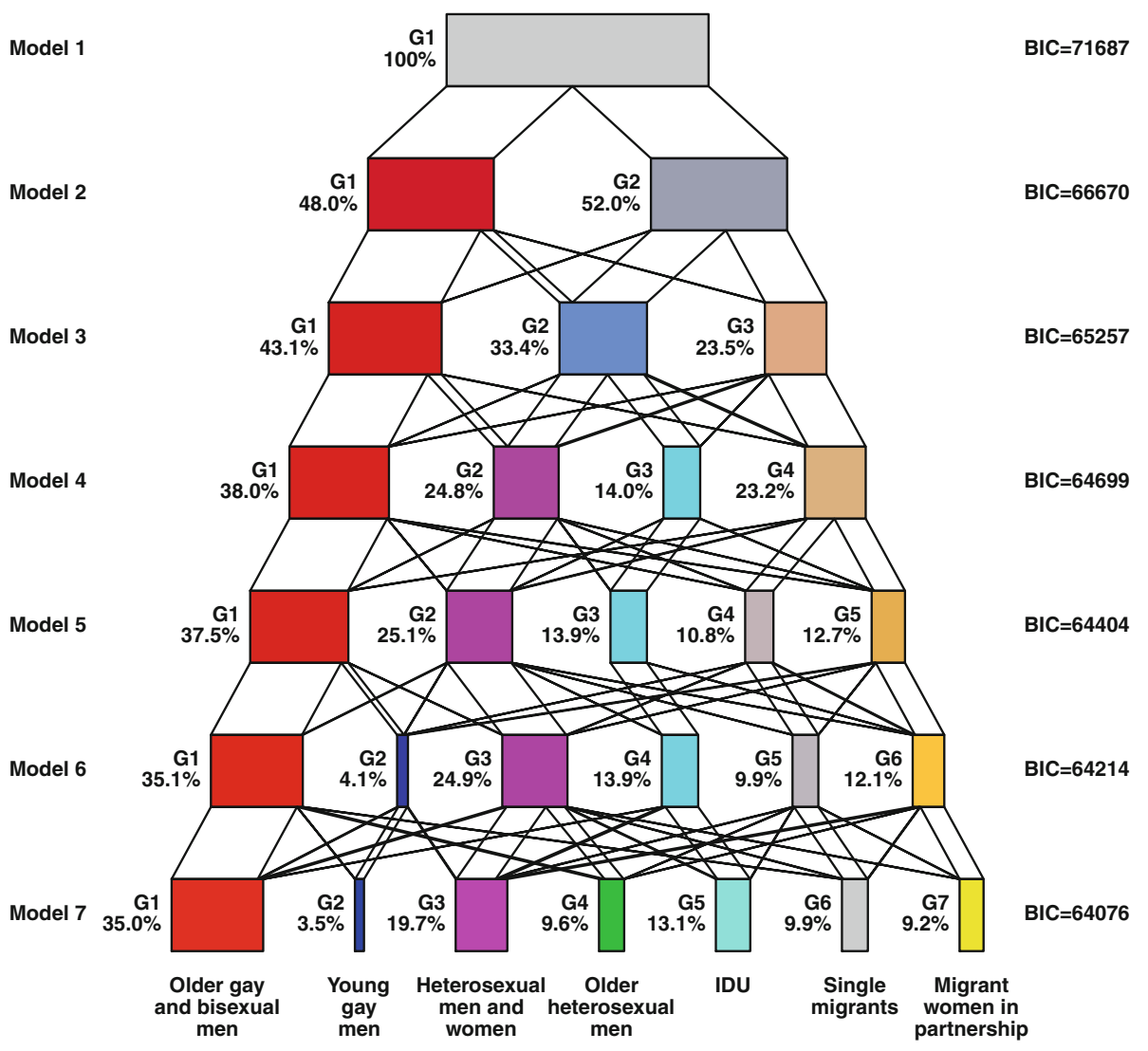

(Kruskal-Wallis $\chi^{2} 24.5, P<0.001$ ). Substantial differences were also observed for the percentage of participants in clinical stage $\mathrm{C}$, which ranged from 8 to $23.9 \%\left(\chi^{2} 17.3\right.$; $P=0.008)$. The prevalence of $\mathrm{HCV}$ infection was highest in IDUs, but also higher in heterosexual men and women and older heterosexual men $\left(\chi^{2} 809.7 ; P<0.0001\right)$ than in other groups. The prevalence of HBV infection was highest in single migrants, migrant women in stable partnerships and younger gay men and lowest in heterosexual men and women. Differences were less pronounced between transmission groups, and failed to reach conventional levels of statistical significance for CD4 cell count and HBV serology.

\section{Outcomes}

A total of 889 participants $(75.3 \%$ of 1,180$)$ suppressed viral load at 6 months, 73 participants $(4.8 \%$ of 1,516$)$ were lost to follow-up and 73 participants $(4.5 \%$ of 1,633$)$ died. Table 3 presents OR or HR for viral suppression, adherence to antiretroviral therapy, loss to follow-up and mortality comparing socio-behavioral groups and transmission groups. In crude and adjusted models the probability of viral suppression six months after therapy start was reduced in IDUs, single migrants and older heterosexual men, compared to older gay and bisexual men.
Similarly, adherence was worse in IDUs and in single migrants. The risk of loss to follow-up was increased in single migrants and IDUs, compared to older gay and bisexual men. Compared to a category combining older gay and bisexual men and younger gay men, mortality was higher in older heterosexual men and IDUs.

Differences were again less pronounced across transmission groups: there were no clear differences in virologic response and participants infected through heterosexual contacts were comparable to gay men with respect to loss to follow-up. Mortality was higher in heterosexually infected participants compared to gay men. As expected, IDUs had a lower probability of virologic response, a higher risk of loss to follow-up and higher mortality, compared to gay men.

\section{Discussion}

We identified several socio-behavioral groups among HIVinfected patients enrolled in the Swiss HIV cohort study through LCA, in support of our first hypothesis. These groups differed from the main HIV transmission groups and include groups that are not directly observable using a single variable approach, thus confirming our second hypothesis. For example, we identified two distinct groups 
Table 1 Distribution of the variables included in the latent class analysis across the seven socio-behavioral groups

\begin{tabular}{|c|c|c|c|c|c|c|c|}
\hline & $\begin{array}{l}\text { Older gay and } \\
\text { bisexual men }\end{array}$ & $\begin{array}{l}\text { Younger gay } \\
\text { and bisexual } \\
\text { men }\end{array}$ & $\begin{array}{l}\text { Heterosexual } \\
\text { men and } \\
\text { women }\end{array}$ & $\begin{array}{l}\text { Older } \\
\text { heterosexual } \\
\text { men }\end{array}$ & $\begin{array}{l}\text { Injection } \\
\text { drug users }\end{array}$ & $\begin{array}{l}\text { Single } \\
\text { migrants }\end{array}$ & $\begin{array}{l}\text { Migrant women } \\
\text { in partnership }\end{array}$ \\
\hline No. of participants & $1568(35.0)$ & $156(3.5)$ & $884(19.7)$ & $431(9.6)$ & $589(13.1)$ & $444(9.9)$ & $411(9.2)$ \\
\hline No. of women & $1(0.1)$ & $5(3.2)$ & $420(47.5)$ & $90(20.9)$ & $202(34.3)$ & $296(66.7)$ & $406(98.8)$ \\
\hline $\begin{array}{l}\text { Median (interquartile range) } \\
\text { age, years }\end{array}$ & $38(33-43)$ & $29(25-34)$ & $37(32-42)$ & $57(52-63)$ & $36(31-41)$ & $30(24-36)$ & $31(27-36)$ \\
\hline \multicolumn{8}{|l|}{ Injection drug use } \\
\hline Never & $1537(98.0)$ & $150(96.2)$ & $780(88.2)$ & 425 (98.6) & $22(3.7)$ & $444(100)$ & $403(98.1)$ \\
\hline Former & $31(2.0)$ & $6(3.9)$ & $104(11.8)$ & $6(1.4)$ & $336(57.1)$ & $0(0)$ & $7(1.7)$ \\
\hline Current & $0(0)$ & $0(0)$ & $0(0)$ & $0(0)$ & $231(39.2)$ & $0(0)$ & $1(0.2)$ \\
\hline \multicolumn{8}{|l|}{ Sexual orientation } \\
\hline Homosexual & $1285(82.0)$ & $130(83.3)$ & $0(0)$ & $74(17.2)$ & $12(2.0)$ & $1(0.2)$ & $3(0.7)$ \\
\hline Bisexual & $174(11.1)$ & $6(3.9)$ & $4(0.5)$ & $22(5.1)$ & $19(3.2)$ & 7 (1.6) & $3(0.7)$ \\
\hline Heterosexual & $107(6.8)$ & $16(10.3)$ & $875(99.0)$ & $328(76.1)$ & $558(94.7)$ & $433(97.5)$ & $405(98.5)$ \\
\hline Unknown & $2(0.1)$ & $4(2.6)$ & $5(0.6)$ & $7(1.6)$ & $0(0.0)$ & $3(0.7)$ & $0(0)$ \\
\hline \multicolumn{8}{|l|}{ Sexual partners } \\
\hline Stable partner only & 354 (22.6) & $92(59.0)$ & $42(4.8)$ & $179(41.5)$ & $206(35.0)$ & $124(27.9)$ & $379(92.2)$ \\
\hline Stable and occasional & $465(29.7)$ & $30(19.2)$ & $668(75.6)$ & $36(8.4)$ & $24(4.1)$ & $5(1.1)$ & $6(1.5)$ \\
\hline Occasional partner only & $603(38.5)$ & $25(16.0)$ & $34(3.9)$ & $51(11.8)$ & $99(16.8)$ & $76(17.1)$ & $0(0)$ \\
\hline No partner & $112(7.1)$ & $6(3.9)$ & $48(5.4)$ & $145(33.6)$ & $217(36.8)$ & $217(48.9)$ & $15(3.7)$ \\
\hline Unknown & $34(2.2)$ & $3(1.9)$ & $92(10.4)$ & $20(4.6)$ & $43(7.3)$ & $22(5.0)$ & $11(2.7)$ \\
\hline \multicolumn{8}{|l|}{ Region of origin } \\
\hline $\begin{array}{l}\text { Switzerland and } \\
\text { North-West Europe }\end{array}$ & $1289(82.2)$ & $45(28.9)$ & $494(55.9)$ & $387(89.8)$ & $476(80.8)$ & $32(7.2)$ & $76(18.5)$ \\
\hline Southern Europe & $130(8.3)$ & $8(5.1)$ & $95(10.8)$ & $35(8.1)$ & $69(11.7)$ & $0(0)$ & $4(1.0)$ \\
\hline Sub-Saharan Africa & $8(0.5)$ & $4(2.6)$ & $144(16.3)$ & $2(0.5)$ & $4(0.7)$ & $399(89.9)$ & $199(48.4)$ \\
\hline Latin America & $51(3.3)$ & $45(28.9)$ & $26(2.9)$ & $0(0)$ & $3(0.5)$ & $2(0.5)$ & $26(6.3)$ \\
\hline Asia, Eastern Europe & $34(2.2)$ & $45(28.9)$ & $89(10.1)$ & $3(0.7)$ & $26(4.4)$ & $5(1.1)$ & $83(20.2)$ \\
\hline Other & $56(3.6)$ & $9(5.8)$ & $36(4.1)$ & $4(0.9)$ & $11(1.9)$ & $4(0.9)$ & $22(5.4)$ \\
\hline \multicolumn{8}{|l|}{ Profession $^{a}$} \\
\hline Self employed & $249(15.9)$ & $16(10.3)$ & $115(13.0)$ & $109(25.3)$ & $25(4.2)$ & $47(10.6)$ & $38(9.3)$ \\
\hline Apprentice/trainee & $5(0.3)$ & $15(9.6)$ & $5(0.6)$ & $0(0)$ & $32(5.4)$ & $15(3.4)$ & $9(2.2)$ \\
\hline Higher management & $134(8.6)$ & $2(1.3)$ & $39(4.4)$ & $33(7.7)$ & $0(0)$ & $0(0)$ & $0(0)$ \\
\hline Middle management & $333(21.2)$ & $2(1.3)$ & $65(7.4)$ & $63(14.6)$ & $25(4.2)$ & $16(3.6)$ & $14(3.4)$ \\
\hline Employee & $835(53.3)$ & $64(41.0)$ & $647(73.2)$ & $202(46.9)$ & $440(74.7)$ & $238(53.6)$ & $142(34.6)$ \\
\hline Houseman/wife & $0(0)$ & $7(4.5)$ & $0(0)$ & $10(2.3)$ & $16(2.7)$ & $39(8.8)$ & $165(40.2)$ \\
\hline Student & $0(0)$ & $44(28.2)$ & $0(0)$ & $0(0)$ & $1(0.2)$ & $23(5.2)$ & $29(7.1)$ \\
\hline Other/unknown & $12(0.8)$ & $6(3.9)$ & $13(1.5)$ & $14(3.3)$ & $50(8.5)$ & $66(14.9)$ & $14(3.4)$ \\
\hline \multicolumn{8}{|l|}{ Main source of income } \\
\hline Work & $1416(90.3)$ & $47(30.1)$ & $756(85.5)$ & $173(40.1)$ & $72(12.2)$ & $58(13.1)$ & $3(0.7)$ \\
\hline Family support & $1(0.1)$ & $93(59.6)$ & $30(3.4)$ & $0(0)$ & $15(2.6)$ & $2(0.5)$ & $361(87.8)$ \\
\hline Insurance/savings & $145(9.3)$ & $13(8.3)$ & $91(10.3)$ & $256(59.4)$ & 494 (83.9) & $377(84.9)$ & $41(10.0)$ \\
\hline Other/unknown & $6(0.4)$ & $3(1.9)$ & $7(0.8)$ & $2(0.5)$ & $8(1.4)$ & 7 (1.6) & $6(1.5)$ \\
\hline
\end{tabular}

Number of participants (\%) are shown unless otherwise indicated. Analysis based on all 4,483 participants enrolled in the Swiss HIV Cohort Study since 1 January 2000

a Current or last held profession

of men infected through sex with men: younger gay men were mainly from Latin America, Asia and Eastern Europe and started ART with a lower median CD4 cell count compared to older gay and bisexual men, who were predominantly Swiss. Most participants who had been infected through heterosexual contacts belonged to one of four 
Table 2 CD4 cell count, clinical stage and prevalence of hepatitis infection at start of ART in socio-behavioral and transmission groups

\begin{tabular}{|c|c|c|c|c|c|c|}
\hline & \multicolumn{3}{|c|}{ CD4 cell count (cells/ $\mu \mathrm{l})$} & \multirow{2}{*}{$\begin{array}{l}\text { Clinical stage } \\
\text { CDC stage C }\end{array}$} & \multicolumn{2}{|c|}{ Hepatitis serology } \\
\hline & $N$ & Median (IQR) & $<200$ cells $/ \mu \mathrm{l}$ & & Hepatitis C & Hepatitis B \\
\hline \multicolumn{7}{|l|}{ Socio-behavioral groups } \\
\hline Older homo- and bisexual men & 630 & $220(146-305)$ & $246 / 581(42.3)$ & $95 / 630(15.1)$ & $25 / 617(4.1)$ & $30 / 595(5.0)$ \\
\hline Younger gay men & 50 & $199(109-264)$ & $22 / 44(50.0)$ & $4 / 50(8.0)$ & $1 / 49(2.0)$ & $4 / 49(8.2)$ \\
\hline Heterosexual men and women & 331 & $175(74-282)$ & $169 / 295(57.3)$ & $71 / 331(21.5)$ & 45/327 (13.8) & $10 / 324(3.1)$ \\
\hline Older heterosexual men & 176 & $219(126-300)$ & $71 / 160(44.4)$ & $42 / 176(23.9)$ & $12 / 176(6.8)$ & $7 / 171(4.1)$ \\
\hline Injection drug users & 174 & $202(108-277)$ & $73 / 150(48.7)$ & 29/174 (16.7) & $148 / 167(88.6)$ & $12 / 172(7.0)$ \\
\hline Single migrants & 123 & $208(116-291)$ & $52 / 108(48.2)$ & 29/123 (23.6) & $5 / 121(4.1)$ & 13/121 (10.7) \\
\hline Migrant women in partnership & 149 & $225(146-330)$ & $49 / 132(37.1)$ & $23 / 149(15.4)$ & $5 / 147(3.4)$ & $12 / 147(8.2)$ \\
\hline$P$ & & $<0.001$ & 0.001 & 0.008 & $<0.001$ & 0.028 \\
\hline \multicolumn{7}{|l|}{ Transmission groups } \\
\hline Men who have sex with men & 648 & $219(147-297)$ & $251 / 593(42.3)$ & 99/648 (15.3) & 24/637 (3.8) & $34 / 613(5.6)$ \\
\hline Heterosexual contact & 748 & $200(106-299)$ & $327 / 670(48.8)$ & $142 / 748(19.0)$ & $56 / 738(7.6)$ & $39 / 733(5.3)$ \\
\hline Injection drug use & 166 & $199(108-277)$ & $73 / 145(50.3)$ & $26 / 166(15.7)$ & $152 / 161(94.4)$ & $11 / 164(6.7)$ \\
\hline Other/unknown & 71 & $202(84-326)$ & $31 / 62(50.0)$ & 26/71 (36.6) & 9/68 (13.2) & $4 / 69(5.8)$ \\
\hline$P$ & & 0.07 & 0.08 & $<0.001$ & $<0.001$ & 0.92 \\
\hline
\end{tabular}

Number of participants (\%) are shown unless otherwise indicated. Analysis based on 1,633 participants who were treatment naïve and started ART at or after enrolment in the Swiss HIV Cohort Study. $P$ values from Kruskal-Wallis tests for continuous variables and $\chi^{2}$ tests for categorical variables

groups: older heterosexual men, single migrants from subSaharan Africa, migrant women from several continents who lived in stable partnerships and a group of heterosexual men and women mainly from Switzerland. Finally, in support of our third hypothesis, we found that group membership was associated with relevant treatment outcomes such as degree of immunodeficiency when starting ART, virologic response to ART, loss to follow-up and mortality. Of note, virologic suppression and adherence was worse and loss to follow-up higher in single migrants from sub-Saharan Africa than in the other groups predominantly infected through heterosexual contacts, and more similar to patients infected through IDU.

\section{Strengths and Weaknesses}

A comparison of cohort data with official AIDS notifications indicated that about $70 \%$ of all individuals living with AIDS in Switzerland participate in the SHCS [25], and a recent comparison with drug sales data showed that $75 \%$ of antiretroviral drug prescriptions in Switzerland can be attributed to study participants [20]. The findings from our study should therefore be applicable to the HIV-infected population in the country, and possibly also to HIV-infected patients starting ART in other industrialized settings.

Our study identified groups that are more informative than the widely used categories of presumed transmission. For example, there were no clear differences across transmission groups in virologic response to ART. Such differences, however, emerged across the groups identified through latent class analysis. Also, the increased mortality (adjusted for age) in heterosexually infected participants is explained by the group of older heterosexual men, and not a general phenomenon. Patients with a history of IDU remain an important risk group, and the inclusion of IDUs largely determined the prevalence of HCV infection in the different groups. In contrast, the prevalence of HBV infection was driven by the region of origin of patients.

LCA is related to other clustering techniques but has a number of advantages. LCA is based on a statistical model and uses objective criteria to identify the optimal number of classes. Furthermore, the method can handle categorical data, accommodates missing values and allows estimating the probability that an individual belongs to a certain group. The decision which variables to include in the model is subjective, however. We selected variables and their categories a priori to avoid bias, and made no changes later on. Results are based on self-reported information on adherence, sexual risk behavior and drug use provided during an interview with a clinician, which is a limitation of our study. Self-reports can be subject to recall bias or social desirability bias. Such bias is, however, probably not substantial: self-reported adherence correlates well with suppression of viral load [26] and longitudinal analyses showed that initiation of IDU was associated with worsening adherence [27].

The information available in the SHCS was rich and covered a wide range of socioeconomic and behavioral 


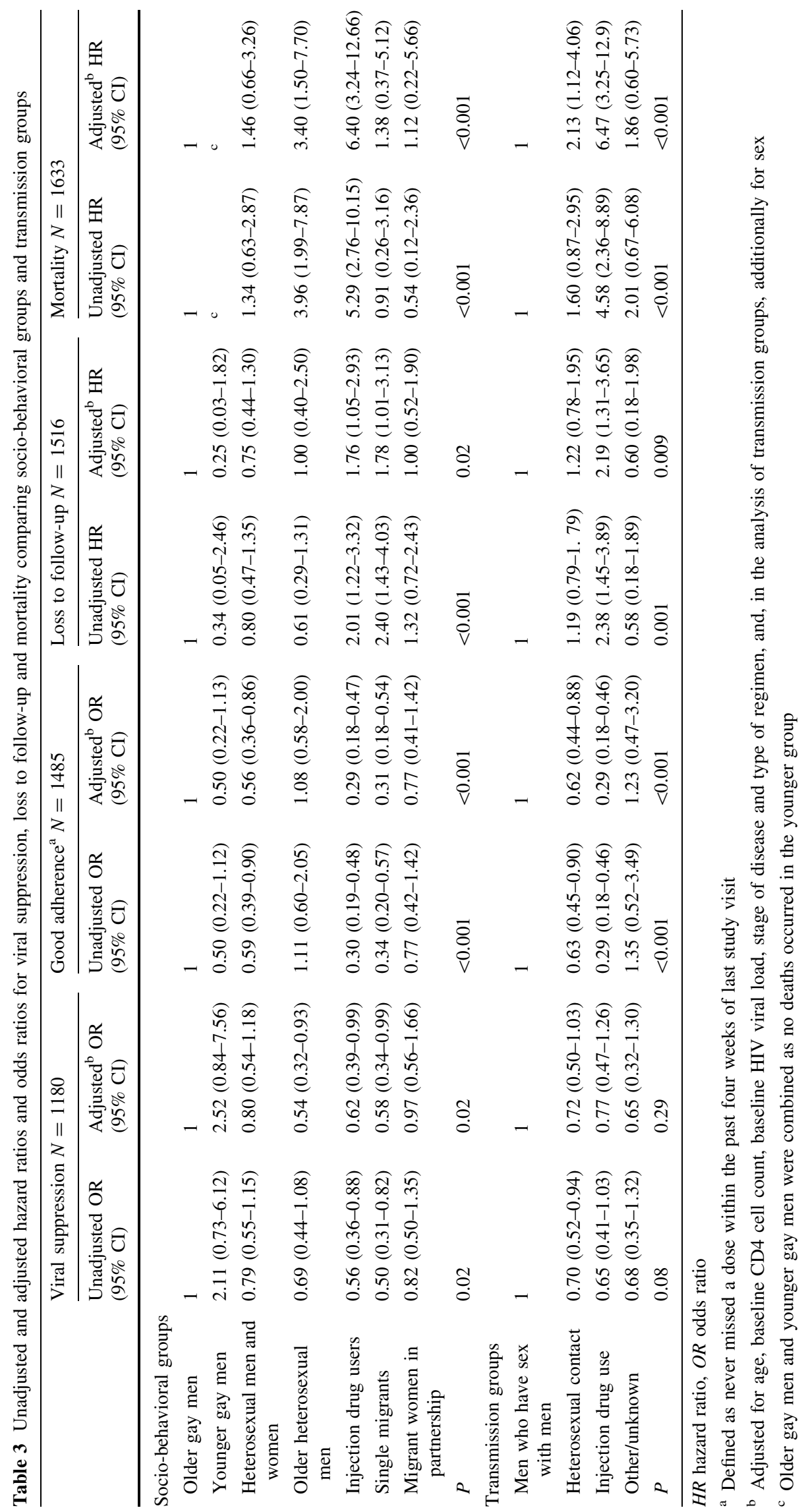


Box 1 Description of socio-behavioral groups

The groups are labelled according to their most pertinent characteristics. Medians (interquartile range) are given for age and the probability of belonging to the group.

1) "Older gay and bisexual men" ( $n=1,568$ participants, $35.0 \%)$

The median age at enrolment was 38 years (33-43 years). The members of this group are mainly from Switzerland and predominantly gay. Many have occasional sexual partners only. They are employed or self-employed. The median probability of belonging to the group was $0.98(0.91-0.99)$.

2) "Younger gay men" ( $n=156$ participants, $3.5 \%)$

The median age was 29 years (25-34 years). The majority is from Latin America, Asia and Eastern Europe. They are employed or students, and the majority have stable partners. Median probability of belonging to the group was $0.96(0.73-1.00)$.

3) "Heterosexual men and women" ( $n=884$ participants, 19.7\%)

The median age was 37 years (32-42 years). The majority is from Switzerland and North-West Europe. They are employed or selfemployed and the majority report a stable and occasional partner. The group includes some individuals with a history of past injection drug use. Median probability of belonging to the group: $0.82(0.63-0.92)$.

4) "Older heterosexual men" ( $n=431,9.6 \%)$

The median age was 57 years (52-63 years). The majority (74\%) are heterosexual men from North-West Europe, but the group also includes some gay men (20\%). Most of them are employees or self-employed, some live from pensions or savings, and many are in stable partnerships. Median probability of belonging to the group: $0.88(0.68-0.96)$.

5) "Injection drug users" $(n=589$ participants, 13.1\%)

The median age was 36 years (31-41) years. Most are former users, about a third report current injection drug use. They are mainly from Switzerland. Many receive support from social insurances. The majority have either stable partners or no partner. Median probability of belonging to the group: 0.99 (0.95-1.00).

6) "Single migrants" $(n=444$ participants, $9.9 \%)$

The median age was 30 years (24-36 years). The majority are heterosexual women and men from sub-Saharan Africa. They are employed or live from social insurance, unemployment benefits, or savings. Two thirds report no partner or occasional partners only. Median probability of belonging to the group: 0.92 (IQR $0.70-0.98$ ).

7) "Migrant women in partnerships" $(n=411,9.2 \%)$

The median age was 31 years (27-36 years). Many are married, some are employed. The majority are from sub-Saharan Africa, Asia and Eastern Europe. Over $90 \%$ live in a stable partnership. Median probability of belonging to the group: 0.97 (IQR 0.91-1.00).

aspects. Groupings remained robust as the number of categories increased, and although groups are not mutually exclusive, membership probabilities were generally high. Since LCA treats all included variables equally, some patients were allocated to groups that are counter-intuitive (for example a few men were classified in the group of migrant women). The mixed group was heterogeneous and median membership probability was low for this group. It is thus not certain that all groups are meaningful and reproducible, and further research into these groups is needed, for example in other populations of HIV-infected patients and through qualitative research.

\section{Methods and Results in Context with Other Literature}

Clinical and epidemiological research is generally concerned with identifying single variables that are associated with the outcome of interest after adjusting for other variables [28]. This approach has also been successful in HIV medicine: the CD4 cell count, for example, is the single strongest risk factor for disease progression, the basis for determining when to start ART [29, 30], and for assessing prognosis [31]. Our study addressed the question whether socio-behavioral variables could help explain the variation of CD4 cell counts at start of ART, and of progression to death. Rather than analyzing the independent contribution of individual socio-behavioral variables, for example the mode of HIV transmission, we identified groups of patients that are similar with respect to many socio-behavioral characteristics. Our approach is therefore more closely related to clinical intuition and experiential clinical reasoning than the usual analysis of individual risk factors [32].

Previous analyses of the Swiss HIV cohort showed that virologic response to ART was poorer in migrants from sub-Saharan Africa [33] and in patients infected through IDU [2]. Another study showed that rates of progression to AIDS or death were lower in patients in stable partnerships compared to other patients [34]. Our results confirm and extend these results: the virologic response in migrants living in stable relationships and levels of adherence were comparable to other sexually infected groups, but poorer in single migrants. Similarly, the socio-behavioral characteristics and outcomes of some former IDUs were closer to the group of heterosexual men and women, than to the group of IDUs, in line with the observation that virologic 
response to ART is better in former IDUs and IDUs attending opiate substitution programmes than in individuals who continue to inject drugs [21]. The group dominated by older heterosexual men also included some gay and bisexual men who were otherwise more similar to this group than to the group of older gay and bisexual men. The prevalence of $\mathrm{HCV}$ infection was higher in the group of older heterosexual men and this group might therefore include some men with a non-disclosed history of IDU. Mortality in this group was increased compared to older gay and bisexual men, even after adjusting for age.

Late diagnosis of HIV and initiation of ART with advanced immunodeficiency is well documented in lowincome settings [35], but also an issue in industrialized countries [36, 37]. For example, a French study found that $36 \%$ of participants were enrolled with a CD4 cell count below 200 cells/ $\mu 1$, or an AIDS defining event [36]. A previous analysis of the SHCS showed that among treatmentnaïve participants with CD4 cell counts above 200 cells/ $\mu 1$, $20 \%$ dropped below this threshold before starting ART [38]. Heterosexual transmission was not associated with late initiation of ART in the previous study. Our analysis indicates that this is an important issue in one group of heterosexually infected patients, but less so in other groups. This could be due to the fact that members of this group and their health care providers do not generally consider them to be 'at risk' and thus not test for HIV as frequently as in other groups.

\section{Implications for ART and Future Research}

Our study has several implications for the provision of ART to different groups of HIV-1 infected patients. Virologic response to ART was worse in migrants with occasional partners or no partners; presumably because of lower levels of adherence to ART. Interventions to increase migrants' ability to adhere to ART should therefore be considered. Although health care insurance is mandatory for all people living in Switzerland and subsidized by the government, some immigrants have a fragile legal status and language barriers and social norms may be a barrier for optimal adherence and care. Other European studies have shown worse treatment outcomes for ethnic minorities and immigrants [39, 40]. Targeted interventions could include culturally adapted adherence counseling, self-help or community groups, general improvement of access to health information and involvement of family members and people who will be trusted by the patient [41].

The reasons for the high rate of loss to follow-up and whether or not ART is continued in patients leaving the cohort should be examined. Interventions to minimize loss to follow-up could include scheduling more frequent follow-up visits in vulnerable groups. Preventing loss to follow-up in immigrants may be more difficult: a previous
SHCS study has shown that many immigrants were asylum seekers and the main reason for leaving the country was the rejection of applications for asylum [42]. The increased mortality in older heterosexual men, which remained after adjusting for age, CD4 cell count and other risk factors, is an intriguing finding: further research into the excess mortality observed in this group is required. Finally, our study highlights the continued need for efforts to improve outcomes in patients infected through IDU, particularly patients who continue to inject drugs.

In conclusion, socio-behavioral groups of HIV-1 infected patients can be identified that differ with respect to the response to ART, adherence, loss to follow-up and mortality. These groups allow insights above what can be gleaned from analyses of the traditional transmission groups and identify patients who may benefit from targeted interventions.

Acknowledgments This study was funded in the framework of the Swiss HIV Cohort Study and a PROSPER fellowhip to O Keiser, both supported by the Swiss National Science Foundation. The members of the Swiss HIV Cohort Study are Barth J, Battegay M, Bernasconi E, Böni J, Bucher HC, Bürgisser P, Burton-Jeangros C, Calmy A, Cavassini M, Dubs R, Egger M, Elzi L, Fehr J, Fischer M, Flepp M, Francioli $\mathrm{P}$ (President of the SHCS), Furrer H (Chairman of the Clinical and Laboratory Committee), Fux CA, Gorgievski M, Günthard $\mathrm{H}$ (Chairman of the Scientific Board), Hasse B, Hirsch HH, Hirschel B, Hösli I, Kahlert C, Kaiser L, Keiser O, Kind C, Klimkait T, Kovari H, Ledergerber B, Martinetti G, Martinez de Tejada B, Müller N, Nadal D, Pantaleo G, Rauch A, Regenass S, Rickenbach M (Head of Data Center), Rudin C (Chairman of the Mother \& Child Substudy), Schmid P,Schultze D, Schöni-Affolter F, Schüpbach J, Speck R, Taffé P, Telenti A, Trkola A, Vernazza P, von Wyl V, Weber R, Yerly S.

\section{References}

1. May MT, Sterne JA, Costagliola D, et al. HIV treatment response and prognosis in Europe and North America in the first decade of highly active antiretroviral therapy: a collaborative analysis. Lancet. 2006;368(9534):451-8.

2. Keiser O, Taffe P, Zwahlen M, et al. All cause mortality in the Swiss HIV Cohort Study from 1990 to 2001 in comparison with the Swiss population. AIDS. 2004;18(13):1835-43.

3. Zwahlen M, Harris R, May M, et al. Mortality of HIV-infected patients starting potent antiretroviral therapy: comparison with the general population in nine industrialized countries. Int J Epidemiol. 2009;38(6):1624-33.

4. Ledergerber B, Egger M, Opravil M, et al. Clinical progression and virological failure on highly active antiretroviral therapy in HIV-1 patients: a prospective cohort study. Lancet. 1999;353(9156):863-8.

5. Pellmar TC, Brandt EN Jr, Baird MA. Health and behavior: the interplay of biological, behavioral, and social influences: summary of an institute of medicine report. Am J Health Promot. 2002;16(4):206-19.

6. Wood E, Hogg RS, Lima VD, et al. Highly active antiretroviral therapy and survival in HIV-infected injection drug users. JAMA. 2008;300(5):550-4.

7. Hinkin $\mathrm{CH}$, Hardy DJ, Mason KI, et al. Medication adherence in HIV-infected adults: effect of patient age, cognitive status, and substance abuse. AIDS. 2004;18(Suppl 1):S19-25. 
8. Appay V, Sauce D. Immune activation and inflammation in HIV-1 infection: causes and consequences. J Pathol. 2008;214(2):231-41.

9. Brenchley JM, Price DA, Schacker TW, et al. Microbial translocation is a cause of systemic immune activation in chronic HIV infection. Nat Med. 2006;12(12):1365-71.

10. McMahon J, Wanke CA, Terrin N, Skinner S, Knox T. Poverty, hunger, education, and residential status impact survival in HIV. Aids Behavior. 2010. (in press).

11. Hogg R, Strathdee SA, Craib KJ, O’Shaughnessy MV, Montaner JS, Schechter MT. Lower socioeconomic status and shorter survival following HIV infection. Lancet. 1994;344(8939):1100-1.

12. Chaisson RE, Keruly JC, Moore RD. Race, sex, drug use, and progression of human immunodeficiency virus disease. $\mathrm{N}$ Engl $\mathrm{J}$ Med. 1995;333(12):751-6.

13. Oramasionwu CU, Brown CM, Lawson KA, Ryan L, Frei CR. Evaluating HIV/AIDS disparities for blacks in the United States: a review of antiretroviral and mortality studies. J Natl Med Assoc. 2009;101(12):122-9.

14. McDavid Harrison K, Ling Q, Song R, Hall HI. County-level socioeconomic status and survival after HIV diagnosis, United States. Ann Epidemiol. 2008;18(12):919-27.

15. Katz MH, Hsu L, Lingo M, Woelffer G, Schwarcz SK. Impact of socioeconomic status on survival with AIDS. Am J Epidemiol. 1998;148(3):282-91.

16. Lazarsfeld R, Henry NW. Latent structure analysis. Latent structure analysis. Boston: Houghton Mifflin; 1968.

17. Spycher BD, Silverman M, Brooke AM, Minder CE, Kuehni CE. Distinguishing phenotypes of childhood wheeze and cough using latent class analysis. Eur Respir J. 2008;31(5):974-81.

18. Ganesalingam J, Stahl D, Wijesekera L, et al. Latent cluster analysis of ALS phenotypes identifies prognostically differing groups. PLoS One. 2009;4(9):e7107.

19. Girardi E, Angeletti C, Puro V, et al. Estimating diagnostic accuracy of tests for latent tuberculosis infection without a gold standard among healthcare workers. Euro Surveill. 2009;14(43):1-9.

20. Schoeni-Affolter F, Ledergerber B, Rickenbach M, et al. Cohort profile: the Swiss HIV Cohort study. Int J Epidemiol. 2010;39(5): 1179-89.

21. Weber R, Huber M, Rickenbach M, et al. Uptake of and virological response to antiretroviral therapy among HIV-infected former and current injecting drug users and persons in an opiate substitution treatment programme: the Swiss HIV Cohort Study. HIV Med. 2009;10(7):407-16.

22. Centers for Disease Control. 1993 revised classification system for HIV infection and expanded surveillance case definition for AIDS among adolescents and adults. MMWR. 1992;41:1-20.

23. McLachlan G, Peel D. Finite mixture models. New York: John Wiley \& Sons; 2000.

24. Rubin D. Multiple imputation for nonresponse in surveys. New York: Wiley; 1987.

25. Gebhardt M, Rickenbach M, Egger M. Impact of antiretroviral combination therapies on AIDS surveillance reports in Switzerland. Swiss HIV Cohort Study. AIDS. 1998;12(10):1195-201.

26. Simoni JM, Kurth AE, Pearson CR, Pantalone DW, Merrill JO, Frick PA. Self-report measures of antiretroviral therapy adherence: a review with recommendations for HIV research and clinical management. AIDS Behav. 2006;10(3):227-45.

27. Glass TR, Battegay M, Cavassini M, et al. Longitudinal analysis of patterns and predictors of changes in self-reported adherence to antiretroviral therapy: Swiss HIV Cohort Study. J Acquir Immune Defic Syndr. 2010;54(2):197-203.

28. Greenland S, Gago-Dominguez M, Castelao JE. The value of riskfactor ("black-box") epidemiology. Epidemiology. 2004;15(5): 529-35.

29. Sterne JA, May M, Costagliola D, et al. Timing of initiation of antiretroviral therapy in AIDS-free HIV-1-infected patients: a collaborative analysis of 18 HIV cohort studies. Lancet. 2009; 373(9672):1352-63.

30. Kitahata MM, Gange SJ, Abraham AG, et al. Effect of early versus deferred antiretroviral therapy for HIV on survival. N Engl J Med. 2009;360(18):1815-26.

31. Egger M, May M, Chene G, et al. Prognosis of HIV-1-infected patients starting highly active antiretroviral therapy: a collaborative analysis of prospective studies. Lancet. 2002;360(9327): 119-29.

32. Braude HD. Clinical intuition versus statistics: different modes of tacit knowledge in clinical epidemiology and evidence-based medicine. Theor Med Bioeth. 2009;30(3):181-98.

33. Staehelin C, Keiser O, Calmy A, et al. Response to antiretroviral therapy in HIV-positive migrants of Sub-Saharan African origin within the Swiss HIV Cohort Study (SHCS). AIDS conference, Mexico 2008. Available at http://www.aids2008.org/Pag/Abstracts. aspx?SID=229\&AID=6062. Accessed 5 May 2010.

34. Young J, De Geest S, Spirig R, et al. Stable partnership and progression to AIDS or death in HIV infected patients receiving highly active antiretroviral therapy: Swiss HIV Cohort Study. BMJ. 2004;328(7430):15.

35. Braitstein P, Brinkhof MWG, Dabis F, et al. Mortality of HIV-1infected patients in the first year of antiretroviral therapy: comparison between low-income and high-income countries. Lancet. 2006;367:817-24.

36. Lanoy E, Mary-Krause M, Tattevin P, et al. Frequency, determinants and consequences of delayed access to care for HIV infection in France. Antivir Ther. 2007;12(1):89-96.

37. Battegay M, Fluckiger U, Hirschel B, Furrer H. Late presentation of HIV-infected individuals. Antivir Ther. 2007;12(6):841-51.

38. Wolbers M, Bucher HC, Furrer H, et al. Delayed diagnosis of HIV infection and late initiation of antiretroviral therapy in the Swiss HIV Cohort Study. HIV Med. 2008;9(6):397-405.

39. Nellen JF, Wit FW, de Wolf F, Jurriaans S, Lange JM, Prins JM. Virologic and immunologic response to highly active antiretroviral therapy in indigenous and nonindigenous HIV-1-infected patients in the Netherlands. J Acquir Immune Defic Syndr. 2004;36:943-50.

40. van den Berg JB, Hak E, Vervoort SC, et al. Increased risk of early virological failure in non-European HIV-1-infected patients in a Dutch cohort on highly active antiretroviral therapy. HIV Med. 2005;6(5):299-306.

41. World Health Organization. National AIDS programme management. Module 4. Targeted HIV prevention and care interventions. 2007.

42. Staehelin C, Egloff N, Rickenbach M, Kopp C, Furrer H. Migrants from sub-Saharan Africa in the Swiss HIV Cohort Study: a single center study of epidemiologic migration-specific and clinical features. AIDS Patient Care STDS. 2004;18(11): $665-75$. 